PRAXIS

ecucativa

Universidad Nacional de La Pampa

Facultad de Ciencias Humanas

Instituto de Ciencias de la Educación

para la investigación interdisciplinaria

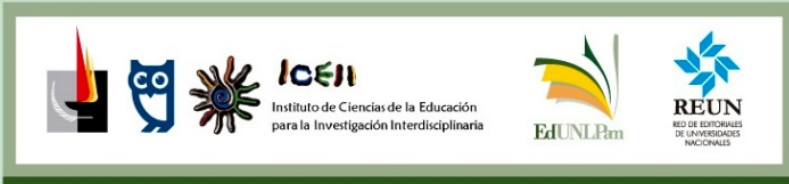

ISSN 2313-934X

SANTA ROSA, LA PAMPA, ARGENTINA

Correo electrónico: iceii@humanas.unlpam.edu.ar

Disponible en https://cerac.unlpam.edu.ar/index.php/praxis

Mecanismos de exclusión social en una escuela preuniversitaria meritocrática. Artículo de Mariano Anderete Schwal. Praxis educativa, Vol 25, No 3 septiembre - diciembre 2021. E - ISSN 2313-934X. pp. 1-20. https://dx.doi.org/10.19137/praxiseducativa-2021-250310

\title{
Mecanismos de exclusión social en una escuela preuniversitaria meritocrática
}

Mechanisms of social exclusion in a meritocratic pre-university school

Mecanismos de exclusão social em uma escola pré-universitária meritocrática

\section{Mariano Anderete Schwal}

Universidad Nacional del Sur

Consejo Nacional de Ciencia y Tecnología, CONICET

Argentina

marianoand3@hotmail.com

ORCID: 0000-0001-5275-3352

Recibido: 2020- 11-14 | Revisado: 2021-04-03 | Aceptado: 2021-05-17 


\section{Resumen}

El artículo parte de la propuesta de modificar el ingreso a una escuela secundaria preuniversitaria tradicional de Bahía Blanca que, desde su creación, sostenía el examen de ingreso. A partir del 2014 se incorporó parcialmente el sorteo y, en 2020, en el contexto del aislamiento social por COVID-19, se impuso el ingreso total por sorteo para los ingresantes externos. El sistema de ingreso meritocrático según los impulsores del cambio, generaría desigualdades y fomentaría la creación de una élite de "clases acomodadas" por parte de la escuela, siendo para ellos el único motivo de la segregación educativa cuestionada. Entonces, el presente trabajo indaga si la escuela dependiente de la UNS tiene mecanismos de inclusión social que atraigan a la matrícula que estaría siendo excluida por el sistema meritocrático o si, por el contrario, refuerza la segregación con distintos mecanismos de exclusión social. Se utiliza una metodología cualitativa basada en entrevistas semiestructuradas a docentes y personal directivo, de la institución analizada y de otras escuelas de la ciudad, complementado con un análisis de la normativa que regula el funcionamiento de la misma, y datos cuantitativos sobre el acceso de sus estudiantes. Se plantea que los mecanismos de inclusión y exclusión exceden al sistema de ingreso propuesto por la universidad y se corresponden con una política educativa de inclusión que se encuentra ausente en la escuela estudiada.

Palabras claves: segregación educativa; meritocracia; escuela preuniversitaria; exclusión social.

\section{Abstract}

The article starts from the proposal to modify the entrance to a traditional pre-university secondary school in Bahía Blanca, which, since its creation, has held the entrance exam. In 2014, the draw was partially incorporated and in 2021, in the context of the quarantine by Covid-19, the total access by draw was imposed for external applicants due to the inequalities of the meritocratic admission system. Which, according to the promoters of change, would promote the creation of an élite of "well-to-do classes" within the school, being for them the only reason for questioned educational segregation. So the present work inquires if the school dependent on the UNS has mechanisms of social inclusion that attract the enrollment that would be being excluded by the meritocratic system, or if, on the contrary, it reinforces segregation with different mechanisms of social exclusion. A qualitative methodology is used based on semi-structured interviews with teachers and principals of the analyzed institution and other schools from its city, complemented with an analysis of the regulations that rule the operation of the school and quantitative data about the access of its students. It is stated that these mechanisms exceed the admission system proposed by the school, and they correspond to an inclusive educational policy that is absent in the school studied.

Keywords: educational segregation; meritocracy; pre-university school; social exclusion.

\section{Resumo}

0 artigo se baseia na proposta de modificar o ingresso em uma escola pré-universitária tradicional de Bahía Blanca, que desde sua criação realiza o vestibular. Em 2014 o sistema de sorteio foi parcialmente incorporado e em 20210, no contexto da quarentena Covid-19, impôs-se o ingresso total por sorteio para os ingressantes externos devido às desigualdades do sistema meritocrático. Sistema que, segundo os promotores da mudança, promoveria a formação de uma elite de "classes abastadas" dentro da escola, sendo para eles o único motivo da questionada segregação educacional. Assim, o presente trabalho investiga se a escola dependente da UNS possui mecanismos de inclusão social que atraiam as matrículas que estariam sendo excluídas pelo sistema meritocrático, ou se, pelo contrário, reforça a segregação com diferentes mecanismos de exclusão social. É utilizada uma metodologia qualitativa a partir de entrevistas semiestruturadas com 
professores e gestores, tanto da instituição analisada quanto de outras escolas da cidade, complementadas com uma análise das normas que regulamentam o funcionamento da escola e dados quantitativos de acesso aos seus alunos. Afirma-se que os referidos mecanismos de inclusão e exclusão extrapolam o regime de admissão proposto pela escola e correspondem a uma política educacional de inclusão que se encontra ausente na escola estudada.

Palavras-chave: segregação educacional; meritocracia; escola pré-universitária; exclusão social.

\section{Introducción}

En el presente estudio, se analiza una escuela secundaria dependiente de la Universidad Nacional del Sur (UNS) de Bahía Blanca, la cual es considerada por la comunidad educativa local como "de élite" por su excelencia académica, lo que la distingue de las demás escuelas públicas locales. Desde su creación en 1962, el sistema de ingreso a la Escuela de Ciclo Básico Común (ECBC) fue por examen, asignando por mérito los cupos disponibles entre los estudiantes de las distintas escuelas públicas y privadas de la ciudad que querían estudiar allí. A partir de 1985, y en el contexto de la vuelta de la democracia, se agregó un curso de nivelación para "igualar las oportunidades" entre los aspirantes. En el año 2013, bajo la premisa de buscar una mayor inclusión social, el Consejo Superior Universitario de la UNS relacionó la meritocracia con el favorecimiento de la segregación educativa y resolvió, a partir del año siguiente, añadir parcialmente el sorteo (50 cupos) al sistema de ingreso, el cual se sumó a los exámenes (158 vacantes) y al acceso directo de quienes provienen de la escuela primaria de la UNS (52 egresados).

El "Ciclo Básico", como se conoce popularmente a la secundaria básica en Bahía Blanca, forma parte de las Escuelas Preuniversitarias de la UNS. Debido a la autonomía universitaria, se trata de escuelas públicas que se diferencian significativamente de las demás escuelas provinciales de la ciudad y cuentan con su propio perfil universitario. El Consejo de Enseñanza Media y Superior (CEMS) es el organismo que coordina a las Escuelas Preuniversitarias de la UNS; no obstante, para realizar modificaciones en el sistema de ingreso, dependen del Consejo Superior Universitario.

Durante el año 2020, el CEMS propuso, en el contexto de la cuarentena impuesta por el COVID-19, quitar el curso de nivelación e imponer el sorteo general para todos los ingresantes externos de la ECBC. La directora del órgano coordinador alegó que el ingreso meritocrático era elitista y significaba la causa principal de la reproducción de la desigualdad por parte de la escuela. La escuela secundaria empezó a ser obligatoria a partir de la Ley de Educación Nacional en 2006 y, por ende, una escuela para todos y todas, no ya para quienes pertenecían a las clases más acomodadas. Por ese motivo, como escuela pública no deberíamos seguir reproduciendo estos dispositivos de selección... Siempre ese examen dio un sesgo de élite a la escuela, mucho más en este momento de profundización de todas las desigualdades en la niñez.

La funcionaria le asigna al examen de ingreso una forma de garantizar el acceso de las

"clases más acomodadas" a la escuela y fomentar una élite educativa, a diferencia de las clases 
"menos acomodadas" que se verían excluidas por tener un peor rendimiento en dicho examen. Entonces, desde el organismo administrativo, propusieron el sorteo general de los ingresantes externos junto con el acceso directo de los egresados de la escuela inicial y primaria de la UNS, donde tienen prioridad los hermanos de los que asisten a la misma escuela y los hijos del personal de la universidad. Asimismo, en el proyecto, solicitaron que estos últimos tengan el privilegio de elegir el turno (mañana o tarde) cuando ingresan directamente, mientras que los demás ingresantes lo obtendrían por azar.

En el presente trabajo, se analizará si, además del sistema de ingreso meritocrático, la escuela lleva adelante mecanismos de inclusión o exclusión de la matrícula por cuestiones socioeconómicas, vinculándose con la segregación educativa que se le atribuye a la secundaria estudiada.

\section{Teoría sobre la segregación educativa, la meritocracia y las escuelas preuniversitarias}

La segregación educativa implica una desigual distribución de estudiantes entre escuelas según su origen, contribuyendo a la reproducción social de la desigualdad ya que los mismos son asignados a circuitos educativos de calidad diferenciada (Krüger, 2019). En este trabajo, se considera la segregación educativa entendida como la división de los estudiantes según su nivel socioeconómico en circuitos educativos desiguales por las experiencias de socialización, condiciones de aprendizaje y saberes que brindan (Veleda, 2014).

Inés Dussel (2014) estudió qué papel juegan las escuelas secundarias en la producción de desigualdades sociales y culturales, las cuales no pasan desapercibidas en dicha temática, sino que juegan un papel clave en el "destino social" de los estudiantes según sus condiciones socioeconómicas de origen. Ni la masificación de la escuela secundaria en Argentina desde mediados del siglo XX ni la obligatoriedad establecida por ley desde el 2006 significaron una modificación de su formato escolar tradicional, caracterizado como selectivo y excluyente (Garino, 2013).

Aguilar (2011) se refiere al concepto de élite como descripción de grupos privilegiados. De acuerdo con Heredia (2005), cabe distinguir los términos de élite y clase alta: mientras que la noción de élite refiere a "los que mandan" y gozan de posiciones de prestigio (De Imaz, 1964), el término "clase alta" se refiere a hijos de familias tradicionales y a ricos de alcurnia vinculados a la tierra (2005, p. 106).

En el libro El colegio, Méndez (2013) analiza el Colegio Nacional de Buenos Aires, considerándolo como un lugar de formación de una élite meritocrática, y a sus miembros como "aristócratas del saber". En los testimonios de los egresados sobre el ingreso, que desde 1957 es mediante un examen eliminatorio, se destaca el fuerte disciplinamiento intelectual (Di Piero, 2016). Méndez señala que los estudiantes tienen orígenes socialmente heterogéneos, pero, una vez dentro, se produce una socialización intensiva cuyos efectos se extienden a sus egresados, quienes se autoperciben como parte de un origen común. El paso por el Colegio Nacional les otorga una identidad en común a sus estudiantes a través de un doble mecanismo: por un lado, hay un 
reconocimiento mutuo entre ellos y, por el otro, se diferencian intelectualmente de quienes no formaron parte de su colegio (Méndez, 2015). En este sentido, la ECBC de la UNS se identifica con el Colegio Nacional de la UBA.

El examen de ingreso es cuestionado por practicar una discriminación hacia aquellos que no tienen el capital cultural suficiente para aprobarlo; Bourdieu se refiere a esta situación como el "racismo de la inteligencia". Asimismo, se lo concibe como un filtro destinado a preservar la naturaleza elitista y diferenciante de algunas instituciones dependientes de Universidades Nacionales que, además su aplicación, garantiza la colonización de la escuela por parte de una clase dominante (Di Piero, 2015). Dubet (2012) señala que la igualdad de oportunidades pretendida por la meritocracia contrasta con la desigualdad de posiciones, por la cual cada uno ocupa su posición social en el sistema educativo y, desde allí, algunas personas están en desventaja para competir con otras.

Con el regreso de la democracia en 1983, se planteó la supresión de los exámenes de ingreso como una medida de "democratización de la educación" por parte del gobierno de Alfonsín, el cual pretendía modificar el sistema educativo autoritario sostenido por la última dictadura argentina (Filmus, 1996). No obstante, las escuelas secundarias preuniversitarias no modificaron automáticamente sus métodos de ingreso, ya que, debido a la autonomía de las Universidades Nacionales, son ellas quienes deciden dichas modificaciones mediante sus órganos de gobierno.

La ECBC de la UNS tomó nota de la democratización del acceso al nivel secundario, entonces, a partir de 1985, incorporó un curso de ingreso voluntario y gratuito para tratar de compensar la "desigualdad de posiciones" que menciona Dubet y, de tal manera, todos los que rendían el examen de ingreso podían tener una base de conocimientos previa aprendidos en la escuela.

Di Piero (2016) reconoce que, en la práctica, la democratización pretendida por el sorteo fue imperfecta. Ya que las escuelas de La Plata que estudió, las cuales tienen un ingreso totalmente por azar desde 1986, continuaban siendo "colonizadas" por familias que transitaron la universidad (tal como sucedía durante el anterior sistema de ingreso por examen) y que dichas instituciones expulsan a quienes no pertenecen a la clase social pretendida mediante proceso de selección "soft". Dicho proceso oculto se corresponde con todas aquellas medidas que tienen las escuelas preuniversitarias para seleccionar a los "alumnos esperados", entre ellas, la autora destaca el capital informacional necesario para inscribirse, los techos de cristal de las familias, el régimen más estricto que en las escuelas provinciales para promocionar el año escolar, la repitencia como predictor de abandono, entre otras medidas sutiles y paulatinas de la institución.

Veleda (2014), al analizar la segregación educativa en la provincia de Buenos Aires, plantea que el sistema de ingreso no es la única causal de segregación educativa (ni la única herramienta para evitarla), sino que la misma se compone de numerosos elementos como la distribución de los alumnos, la designación los docentes, los recursos materiales y las distintas ofertas pedagógicas entre las escuelas. 
En la presente investigación, se analiza a una escuela secundaria que, junto con el resto de las Escuelas Preuniversitarias de la UNS, se identifica con las instituciones tradicionales del país y se destaca como parte de una élite educativa en la ciudad de Bahía Blanca: atiende mayoritariamente a sectores sociales que asistieron a la universidad o aspiran a transitarla y los principales actores de la escuela consideran que la misma ocupa un lugar de vanguardia pedagógica que se anticipa y se diferencia de los demás establecimientos educativos de la ciudad. En tal sentido, se intentará demostrar si el sistema de ingreso meritocrático funciona como el único mecanismo de exclusión social en la ECBC o si, por el contrario, la escuela fomenta diversas prácticas que alejan a un sector socioeconómico determinado. En el trabajo, se considerará si la UNS, a través de sus escuelas dependientes, desarrolla políticas educativas de inclusión social como lo hacen otras escuelas preuniversitarias del país.

A fines del año 2013, el Ministerio de Educación lanzó un "Proyecto de creación de Nuevas Escuelas Secundarias con Universidades Nacionales" (PCNESUN), por el cual distintas Universidades Nacionales se involucraron en la creación de nuevas escuelas secundarias que tenían por objeto incluir a jóvenes en situación de desigualdad social y educativa. El rasgo más destacado es la propuesta de orientar la escuela a la atención de jóvenes que vivan en barrios vulnerables, desafiando el supuesto destino de exclusión educativa y social, lo que remite a la posibilidad de pensar en políticas de discriminación positiva a cargo de las Universidades Nacionales. La diferencia con las escuelas preuniversitarias tradicionales (como la estudiada en el presente trabajo) es que las nuevas no estén destinadas a los estudios académicos de una élite, sino que comprenden a sectores populares y su objetivo principal es la inclusión social con calidad educativa (Antico et al., 2019). Sus políticas de inclusión social exceden ampliamente el método de ingreso, el cual varía entre las distintas escuelas creadas (Anderete Schwal, 2021), realizando políticas activas hacia los estudiantes que están socialmente relegados. No obstante, cabe destacar que dichos colegios proponen la inclusión mediante una matrícula socialmente homogénea, compuesta exclusivamente por jóvenes provenientes de sectores de bajos recursos.

\section{Metodología}

A los fines de determinar el objetivo planteado, se realiza un análisis cualitativo con base en entrevistas semiestructuradas a docentes y personal directivo de la ECBC respecto de las temáticas planteadas. También se incluyen testimonios de directivos de escuelas públicas de la ciudad. Asimismo, se coteja con la normativa de la UNS respecto de sus escuelas dependientes y se presentan datos cuantitativos sobre el acceso de estudiantes con diversos métodos de ingreso.

Las entrevistas a los docentes y las directoras de la escuela aportan perspectivas valiosas para comprender los distintos mecanismos de inclusión o exclusión que reproduce la institución educativa. También se suman los puntos de vista de directoras de escuelas públicas ubicadas en la periferia pobre de la ciudad y cuyos estudiantes no eligen la escuela secundaria de la UNS, a modo de tener otra perspectiva sobre la exclusión. 
Al analizar este método de investigación, Dussel (2009) menciona como limitación intrínseca la comprobada tendencia de los entrevistados a declarar como propias opiniones que creen convenientes y compartidas por la mayoría. Este fenómeno es conocido como "deseabilidad social" (Corbetta, 2003), el cual explica posiblemente parte de la apreciación personal al referirse a instituciones tan valoradas socialmente como la escuela. En el caso de los directivos, además, acostumbrados a asumirse como "voz oficial" de la escuela, como portavoces ante la comunidad escolar y ante la sociedad, el efecto de deseabilidad podría pronunciarse (Dussel, 2014).

A continuación, en base al análisis normativo de la UNS junto con las declaraciones de docentes y directivos, se presentan los diversos mecanismos de exclusión social llevados adelante por la escuela.

\section{Los mecanismos de exclusión en la ECBC}

Seguidamente, se desarrollan las distintas formas de exclusión que se dan en la escuela estudiada, según la reglamentación de la institución, datos cuantitativos y los testimonios de docentes y directivos.

\section{Poca inclusión social}

Los trabajadores entrevistados de la ECBC coinciden al mencionar que sus estudiantes pertenecen a sectores de clase media o media alta, quienes tienen necesidades básicas satisfechas y no sufren los problemas económicos que se pueden observar en otras escuelas públicas de provincia. "En general, los alumnos (del Ciclo Básico) son de clase media alta. Tienen muchos viajes en el año, no tienen ningún tipo de dificultad económica, ni para conseguir libros o fotocopias" (Profesor de Lengua 2, ECBC, 2019).

La Escuela no se caracteriza por recibir alumnos de bajo nivel socioeconómico. No obstante, se observan unos pocos casos de estudiantes que reciben ayuda para gastos de fotocopias.

En la escuela, hay muy pocos becados, te podría decir que cuatro de una población de 700 alumnos. Solamente tuvimos un caso de un alumno que utilizaba el comedor de la escuela, en la cantina de las EMUNS siempre le preparaban el almuerzo. Aunque cuando pasó al Ciclo Básico ya no comió en la escuela porque dijo que le daba vergüenza. (Directiva, EElyP, UNS, 2019)

A continuación, se analiza la inscripción de estudiantes provenientes de escuelas ubicadas en la periferia pobre de la ciudad (Chávez, 2017), entendida como aquella donde se encuentran los sectores de clases bajas, donde la mayor parte de los jóvenes desarrollan su trayectoria educativa y sus vínculos sociales en sus propios barrios (Veleda et al., 2011). El objetivo del sorteo sería atraer a dicha población. Pero, en la tabla propuesta a continuación, se observa la evolución de la inscripción de egresados de la primaria a medida que fue cambiando el sistema de ingreso, partiendo del meritocrático puro, pasando por uno mixto y finalizando con el sorteo total, tal como sucedió en el ingreso del ciclo lectivo 2021 por el contexto de la pandemia. 


\section{Cuadro 1}

Inscriptos a la ECBC provenientes de escuelas públicas ubicadas en la periferia pobre de Bahía Blanca: años 2013, 2016, 2018 y 2021

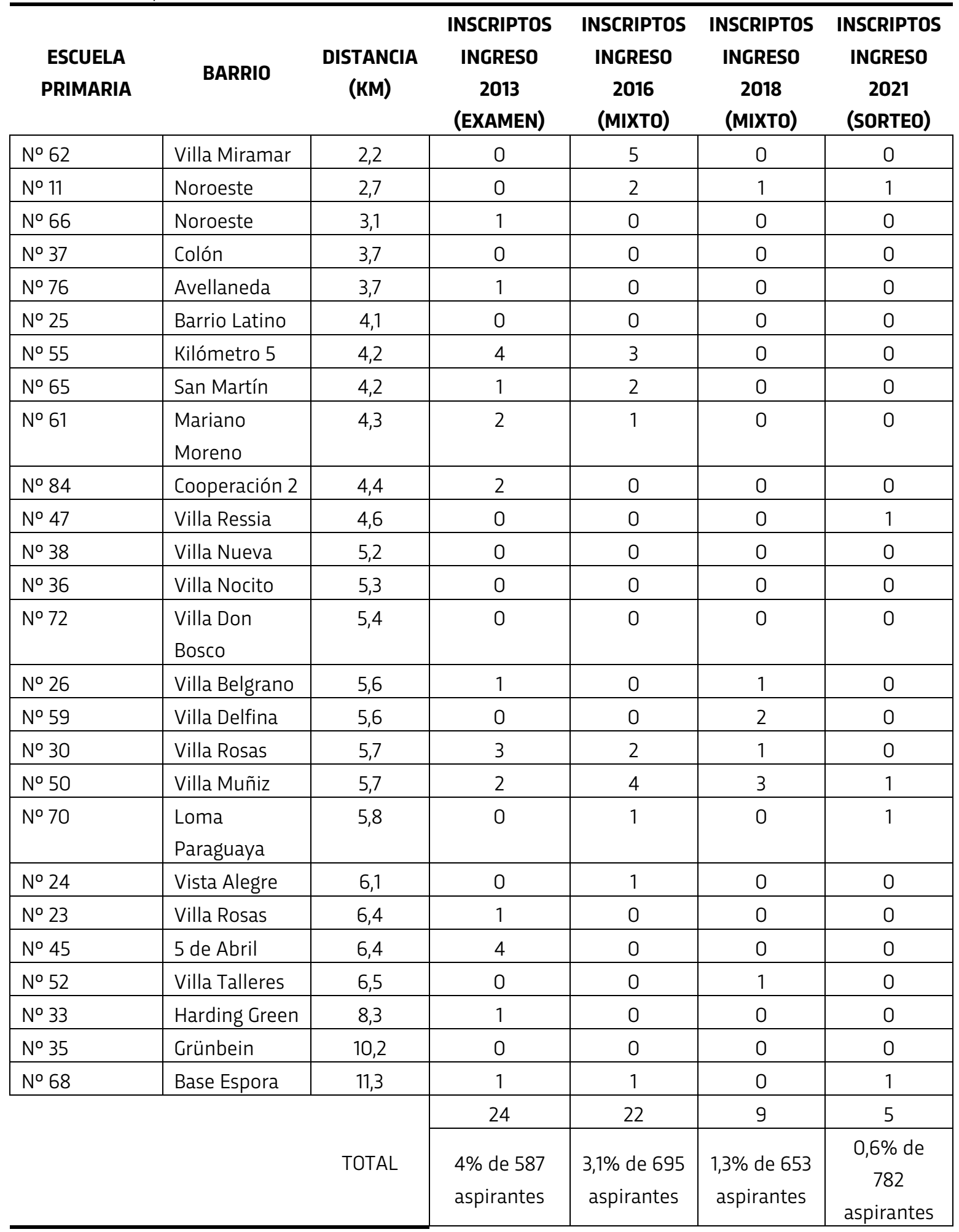


Al analizar el Cuadro 1, se observa que la disminución de alumnos inscriptos provenientes de escuelas públicas de la periferia es constante desde el año 2013, que fue el último año con ingreso meritocrático total. A partir del año 2014, se incorporó parcialmente el sorteo con la promesa de lograr una escuela más heterogénea socialmente y atraer a dichos sectores, ii pero, con el paso de los años, la convocatoria parece tornarse socialmente más excluyente. Finalmente, en el año 2021, se logró el ingreso total por sorteo, aunque el interés de dichos sectores es el más bajo de los últimos años. La explicación a estos resultados cuantitativos se encuentra en la falta de políticas educativas inclusivas por parte del CEMS y de la UNS.

\section{Información sobre el ingreso}

La exclusión de un sector social en la ECBC se debe no solo a cuestiones económicas y de aspiraciones académicas, sino también a la falta de información. En este sentido, se indaga sobre la divulgación que realiza la ECBC de su oferta educativa, consistente en pegar cartelería en el ha// de la escuela y en enviar un correo electrónico anualmente al Consejo Escolar y a la Dirección de Educación de Gestión Privada (DIPRECEP) de Bahía Blanca para que sea reenviado a las instituciones educativas públicas y privadas de la ciudad. Esa es la única comunicación que tiene con el resto de las escuelas. Se pretende atraer un público determinado de instituciones educativas de la periferia, pero ni siquiera se tiene un contacto directo con ellas.

Todos los años, cuando se publica el ingreso, les mandamos mails al consejo escolar y al DIPREGEP, ellos se encargan de reenviarlos a todas las escuelas. Además, se publica en la página de la escuela y pegamos carteles en el hall de entrada y en algunos departamentos de la UNS también se pegan. (Directiva, ECBC, 2018)

Respecto de la difusión de la inscripción a la ECBC, el Artículo 10 del CSU 283/14 dice:

En el transcurso del mes de abril, se realizará la difusión en las E.M.U.N.S. y, a través de la Dirección de Prensa y Ceremonial de la U.N.S., en los medios masivos de comunicación y en la página Web de la U.N.S.

Pero no se observa registro alguno en medios masivos de comunicación locales que se refieran a la difusión de la inscripción, mientras que sí se observa difusión en la cartelería de las EPUNS (escuelas preuniversitariasde la UNS) y en la sección noticias del portal web oficial de la UNS. iii Sin embargo, dichos lugares son frecuentados por gente vinculada con la universidad y no tiene un alcance a la población en general. De tal forma, son siempre los mismos sectores sociales los que reciben la información sobre el ingreso a la ECBC. La difusión de la oferta educativa existe, pero parece estar orientada a un público determinado.

Las familias no se enteran de cómo es el ingreso al Ciclo Básico, pero tampoco están interesadas en mandar a sus hijos. A veces vemos algún chico que se destaca y le decimos a sus familiares que podría anotarse en la escuela de la universidad, pero lo ven como algo muy lejano y no conocen a nadie que haya ido. Así que prefieren no anotarlo. (Directora primaria pública, Barrio Latino, 2019) 
"La verdad es que, al no conocerla, uno no pide lo que no conoce" (Directora primaria pública, Bella Vista, 2019). Las directoras de las escuelas de la periferia se refieren al desconocimiento que tienen las familias de sus barrios sobre la oferta de la escuela preuniversitaria. La difusión anual sobre el ingreso no alcanza a dichos sectores, quienes no conocen la oferta educativa preuniversitaria y, por tal motivo, no la consideran como una posibilidad educativa para sus hijos.

\section{Falta de inclusión dentro de la comunidad educativa local}

En cuanto a la integración de la ECBC con el resto de las escuelas de la ciudad, cabe destacar que no existe ningún programa de extensión para sus docentes (Landivar, 2019), por lo cual la institución preuniversitaria no llega a los barrios ni a las escuelas de la periferia.

La escuela de Ciclo Básico no se relaciona con otras escuelas, se relaciona con algunas instituciones que tienen que ver con abuelos, con la cooperativa, con la Universidad... pero no tiene ninguna relación con otras escuelas. En primaria, vamos a hacer un trabajo de extensión, porque nos invitaron, con una escuela rural de Cerri. Los chicos van a venir acá y nuestros chicos van a ir allá, y se terminó la actividad. Es la primera vez en años.

¿Por qué no vienen de esos sectores (periféricos) a la escuela? Porque tampoco vamos nosotros, porque no nos mostramos interesados. (Directiva de la ECBC, 2019)

La ECBC no se vincula con otras escuelas de la ciudad, sino que actúa como una institución que brinda alta calidad educativa, pero aislada del resto del sistema educativo de la ciudad. Esta situación contrasta con el ejemplo de la Escuela Ernesto Sábato de la UNICEN (Tandil) que, dentro de sus objetivos, ${ }^{\text {iv }}$ sostiene:

Generar acciones de articulación con la totalidad del sistema educativo regional y local, de diferentes gestiones (estatal y privada) y categorización de las instituciones educativas (geográficas, socioculturales y de matrícula) en torno a los ejes de capacitación, desarrollo de proyectos especiales y experiencias innovadoras. (2014)

De tal manera, se logra una integración de la escuela preuniversitaria en el campo educativo local y se pueden alcanzar algunos objetivos de inclusión y calidad educativa. A su vez, en la página web oficial de la UNSAM, una publicación ${ }^{\vee}$ da cuenta de la labor socialmente inclusiva que desarrollan los docentes de la escuela, donde salen a recorrer el barrio para contarles a las familias la propuesta educativa de la escuela y, de tal forma, invitar a los jóvenes a inscribirse o continuar en la institución.

Unos días antes de empezar las clases, los maestros de la Escuela Secundaria Técnica de la UNSAM recorren los barrios del área Reconquista, visitan los hogares de sus estudiantes y organizan convivencias en la Escuela. Son acciones que amplían la inclusión y fortalecen el vínculo con el territorio. (...) Los profesores organizan por grupos, llevan un mapita y un listado de los hogares que van a visitar. A veces no es fácil encontrar las casas. Nos recibe una mamá o un papá, nos acercan sillas y nos ofrecen algo para tomar, a veces con bebés a 
upa y varios hermanitos dando vueltas. Entonces los profes hablan con el familiar a cargo y con el/la adolescente que va a ingresar o vuelve la escuela. (2020)

Se aprecia el esfuerzo extra de los profesores para convocar al alumnado del barrio, teniendo en consideración sus características socioeconómicas y familiares.

\section{Exclusión de personas con discapacidad}

Respecto a la inclusión de personas con discapacidad, el Ciclo Básico no se caracteriza por tener alumnos integrados. "Hay personas (con discapacidad) en inicial o primaria, pero no se anotan en el Ciclo Básico. Tendrían que rendir como todo el mundo, pero no se anotan" (Directiva de la ECBC, 2019).

El sistema de ingreso cerrado en primer año del secundario atenta contra la Resolución 311/16 del Consejo Federal de Educación, la cual dispone la obligatoriedad a todas las primarias y secundarias de recibir estudiantes con discapacidad provenientes de escuelas especiales. Si una persona con discapacidad quisiera ingresar a la ECBC, debería anotarse para el ingreso al primer año y beneficiarse con el sorteo parcial o aprobar los exámenes de ingreso, a diferencia de las demás escuelas públicas provinciales que tienen cupo todos los años para recibir estudiantes con discapacidad. No obstante, no se registran casos en la ECBC ya que la escuela no se caracteriza por favorecer activamente este tipo de inclusión.

Desde el punto de vista edilicio, la escuela cuenta con un ascensor para cubrir las necesidades especiales de quienes no puedan subir las escaleras (la ECBC funciona en el primer y segundo piso del edificio central de las EPUNS). Sin embargo, hay muy pocos casos de estudiantes con discapacidades físicas en los últimos años, y el uso del ascensor se reduce a una incapacidad temporal de los estudiantes (por ejemplo, el caso de quebraduras). Asimismo, la escuela cuenta con recursos audiovisuales en la biblioteca para poder cubrir otro tipo de discapacidades; no obstante, no son utilizadas.

Los docentes no están preparados para trabajar con la diversidad, para poder incluir alumnos con discapacidad se necesitarían generar cargos pedagógicos y capacitar a los docentes. Hay escuelas privadas que están preparadas para la inclusión y lo hacen muy bien, pero no es el caso del Ciclo Básico. (Directiva de la ECBC, 2019).

La ECBC no acostumbra a recibir estudiantes con discapacidad, por lo cual su integración significaría un desafío para los profesores que están acostumbrados a trabajar con otro tipo de estudiantes. Respecto de las dificultades cognitivas, anualmente se registran pocos ingresantes con Trastorno por Déficit de Atención e Hiperactividad (TDHA), lo cual no constituye una discapacidad, pero sí un trastorno en el aprendizaje. En las entrevistas, los docentes hablan sobre la falta de preparación para la atención de este tipo de estudiantes, quienes no ingresan por examen, sino que los pocos casos continúan desde la primaria de la UNS o salen sorteados. No obstante, la incorporación de alumnos con problemas cognitivos fue mínima desde la inclusión del sorteo porque las familias siguen teniendo en cuenta la exigencia académica como el motivo principal al 
momento de la inscripción de sus hijos, y no la inclusión educativa de quienes tienen otros tiempos para el aprendizaje.

\section{Lenguaje "inclusivo"}

La inclusión en la escuela se observa desde el punto de vista del "lenguaje inclusivo" en cuanto al género de las personas, vi ya que la escuela permite la utilización del mismo en la documentación curricular, en la comunicación institucional y en el interior de las aulas. Las EPUNS aprobaron esta normativa antes que la UNS vii debido a una política activa en la materia por parte de las autoridades del CEMS. Esto significa que los profesores y los estudiantes pueden utilizar la letra "x", el símbolo "@" o la letra "e" al referirse a un sujeto plural por escrito y oralmente (cuando lo permita el principio de oralidad del idioma castellano). Gil (2019) indica que este es un reclamo planificado, no un cambio espontáneo que proviene de los hablantes de los sectores sociales más empobrecidos, sino que es estimulado por círculos académicos e intelectuales. El autor señala que una paradoja de este "lenguaje" es que se piensa en un cambio para toda la sociedad, sin embargo, es un cambio sostenido y articulado por personas altamente escolarizadas y con conciencia gramatical, que no representan necesariamente el habla de los sectores sociales populares. Razón por la cual el "lenguaje inclusivo" termina siendo paradójicamente elitista.

El lenguaje inclusivo fue aprobado por el CEMS a pedido de un grupo de profesoras de lengua que lo usan, después lo fueron sumando algunas profesoras más del área de ciencias sociales. Hay alumnas que también lo usan en clase, siempre se relaciona con reclamos políticos del feminismo. (Profesor de Lengua 2 del ECBC, 2019).

El lenguaje inclusivo permite visibilizar las imposiciones machistas de la lengua que utilizamos diariamente, fue una conquista por parte de quienes formamos parte de las EMUNS que se aceptara su uso, incluso antes de que lo haga la UNS. Es voluntario, por lo tanto, la persona que se sienta identificada podrá usarlo. Hoy en día, hay muches alumnes que lo usan. (Profesora de Historia, ECBC, 2019)

Bernstein (1988) elaboró la teoría de los códigos lingüísticos de las distintas clases sociales, asociados a las pautas socializantes vigentes en familias de distinto origen social. Los códigos son las formas de discurso presentes durante los primeros años de vida del niño, que luego afectan su experiencia escolar posterior. El autor distingue entre el código lingüístico elaborado y el código restringido; las diferencias entre ambos se refieren tanto a los aspectos de vocabulario como a las estructuras de significado que operan en cada uno de los códigos.

La ECBC se encuentra más adaptada a aquellos alumnos que tienen un código lingüístico elaborado, correspondiente a un sector con determinada base cultural que está dispuesta a cambiar gramaticalmente el lenguaje, aunque en la práctica de dicha escuela el "lenguaje inclusivo" solamente es utilizado por un sector de los docentes y estudiantes que sostienen una postura política determinada y no es empleado por la mayoría de los actores educativos de la institución.

Tuvimos una maestra suplente que una vez les escribió a los chicos con lenguaje inclusivo, al otro día teníamos una madre quejándose en la dirección. En las escuelas de la 
universidad, deben tener otros códigos, pero acá a los chicos no están acostumbrados a usarlo y a algunas familias les genera rechazo. (Directora Primaria Pública, Barrio Avellaneda, 2019).

Mientras que, en los colegios de la UNS, está reglamentado, en las demás escuelas públicas provinciales el lenguaje inclusivo no está reconocido oficialmente por las autoridades educativas. Asimismo, de acuerdo con lo indicado por las directoras, se advierte que no es utilizado por los estudiantes y los docentes que pretenden aplicarlo reciben resistencia por parte de las familias.

\section{Los herederos de la UNS}

El cupo para los hijos del personal de la UNS parece una nueva versión —-más explícita — de Los Herederos de Bourdieu y Passeron (2003), por cuanto pueden formar parte de la educación inicial y primaria de la UNS preferentemente aquellos que sean familiares de quienes pertenecen actualmente a la institución (con determinado capital económico, social y cultural). Posteriormente, estos alumnos ingresan directamente a la ECBC. De esta forma, se aseguran la socialización entre pares de la misma clase social, por parte de quienes forman parte de la comunidad universitaria. Dicho sistema de ingreso no es el histórico en el nivel inicial, sino que fue una conquista del gremio docente de la UNS obtenida a mediados de los 90. El CEMS, al proponer el sorteo total en el año 2020, no reclamó la modificación de este tipo de ingreso elitista, sino que reafirmó sus privilegios proponiendo que los ingresantes directos a la ECBC sean los únicos que puedan elegir el turno donde cursarán su primer año de secundaria, a diferencia del resto que se le asigna por sorteo.

\section{Cuadro 2}

Delegación municipal de residencia de los ingresantes 2021 de la ECBC

\begin{tabular}{|c|c|c|c|c|c|c|c|}
\hline \multirow[t]{2}{*}{$\begin{array}{c}\text { Delegación municipal } \\
\text { de residencia }\end{array}$} & \multirow{2}{*}{\begin{tabular}{|c} 
Total Censo \\
2010
\end{tabular}} & \multicolumn{2}{|c|}{$\begin{array}{c}\text { Sorteados } \\
\text { Escuelas públicas }\end{array}$} & \multicolumn{2}{|c|}{$\begin{array}{c}\text { Sorteados } \\
\text { Escuelas privadas }\end{array}$} & \multicolumn{2}{|c|}{$\begin{array}{l}\text { Ingresantes } \\
\text { Directos } \\
\text { Primaria UNS }\end{array}$} \\
\hline & & Ingresantes & $\%$ & Ingresantes & $\%$ & Aspirantes & $\%$ \\
\hline CENTRO & 35,12 & 39 & 28,26 & 44 & 36,67 & 31 & 59,62 \\
\hline LAS VILLAS & 17,12 & 13 & 9,42 & 14 & 11,67 & 7 & 13,46 \\
\hline NORTE & \multirow{2}{*}{$15,30^{*}$} & 21 & 15,22 & 20 & 16,67 & 3 & 5,77 \\
\hline SESQUICENTENARIO & & 4 & 2,90 & 13 & 10,83 & 4 & 7,69 \\
\hline VILLA ROSAS & 10,63 & 19 & 13,77 & 11 & 9,17 & 1 & 1,92 \\
\hline NOROESTE & 10,6 & 12 & 8,70 & 3 & 2,50 & 2 & 3,85 \\
\hline INGENIERO WHITE & 4,14 & 3 & 2,17 & 1 & 0,83 & 0 & 0,00 \\
\hline HARDING GREEN & 3,66 & 17 & 12,32 & 12 & 10,00 & 4 & 7,69 \\
\hline GENERAL CERRI & 2,72 & 10 & 7,25 & 1 & 0,83 & 0 & 0,00 \\
\hline \multirow[t]{2}{*}{ CABILDO/OTRO } & 0,67 & 0 & 0,00 & 1 & 0,83 & 0 & 0,0 \\
\hline & 100 & 138 & 100 & 120 & 100 & 52 & 100 \\
\hline
\end{tabular}

*En 2020, la delegación Norte se dividió en dos, creando la delegación Sesquicentenario 
En el Cuadro 2, se analiza el origen residencial de los ingresantes (52 directos de la primaria UNS y 158 sorteados de otras escuelas), destacándose que aquellos provenientes de la propia universidad —quienes prioritariamente son descendientes del personal universitario—residen mayoritariamente en la delegación centro de la ciudad, siendo la que mejores índices de calidad de vida representa. Son familias con estabilidad económica y con cierto capital cultural, configurando una elite universitaria que tiene su propio método de ingreso. Registran un origen socioeconómico mayor y más homogéneo que aquellos provenientes de escuelas privadas. Esto se debe a la socialización controlada que tienen los ingresantes directos, pertenecientes mayoritariamente a familias universitarias. Por otra parte, los ingresantes externos representan una distribución residencial diferente, de acuerdo con el sector público o privado de su escuela primaria de origen. Aquellos provenientes de escuelas públicas registran una mayor heterogeneidad social y más próxima a la distribución arrojada por el último censo practicado en la ciudad.

\section{Inc/usión meritocrática}

El sistema de ingreso por exámenes consiste en admitir a quienes tienen mayores méritos académicos, incluyendo solamente a los estudiantes de grupos sociales desfavorecidos que se destaquen en las pruebas de selección. Ellos, quienes compiten sin ningún tipo de ventaja o consideración respecto de los demás. Bourdieu y Passeron (2003) los Ilaman "los becarios" (2003), se ganan un lugar a base de esfuerzo.

Las posibilidades de estudio de aquellos que tienen una buena situación económica no es la misma de quienes tienen dificultades sociales, económicas y familiares, ellos deben superar obstáculos mayores que los primeros para obtener los mismos resultados educativos (Langer y Esses, 2019). El examen de ingreso no tiene en cuenta estas diferencias y trata a todos por igual, tornándose inequitativo para aquellos con mayores dificultades educativas de origen. "No tiene sentido la dificultad en el examen del Ciclo Básico, porque son conocimientos que se exigen en la secundaria y no en sexto grado. Ni siquiera los que van a la primaria del Ciclo aprueban esos exámenes" (Directora Primaria Pública, Barrio Bella Vista, 2019).

La crítica recurrente al examen de ingreso es su dificultad, puesto que requiere de una preparación especial por parte de los aspirantes para tener mayores chances en el ingreso.

\section{Cuadro 3}

Preparación para el examen de ingreso 2019 de la ECBC según escuela de origen

\begin{tabular}{|c|c|c|c|c|c|c|c|c|c|c|c|}
\hline $\begin{array}{l}\text { Escuela primaria } \\
\text { de origen }\end{array}$ & $\begin{array}{l}\text { Total } \\
\text { alumnos }\end{array}$ & \multicolumn{5}{|c|}{ Estudió con profesor particular } & \multicolumn{5}{|c|}{ No estudió con profesor particular } \\
\hline \multirow[b]{2}{*}{ Pública } & \multirow[b]{2}{*}{282} & \multirow[b]{2}{*}{$80,8 \%$} & \multirow[b]{2}{*}{228} & Aprobó & $41,7 \%$ & 95 & \multirow[b]{2}{*}{$19,2 \%$} & \multirow[b]{2}{*}{54} & Aprobó & $31,5 \%$ & 17 \\
\hline & & & & Desaprobó & $58,3 \%$ & 133 & & & Desaprobó & $68,5 \%$ & 37 \\
\hline \multirow[b]{2}{*}{ Privada } & \multirow[b]{2}{*}{170} & \multirow[b]{2}{*}{$73,5 \%$} & \multirow[b]{2}{*}{125} & Aprobó & $63,2 \%$ & 79 & \multirow[b]{2}{*}{$26,5 \%$} & \multirow[b]{2}{*}{45} & Aprobó & $33,3 \%$ & 15 \\
\hline & & & & Desaprobó & $36,8 \%$ & 46 & & & Desaprobó & $66,7 \%$ & 30 \\
\hline \multirow[b]{2}{*}{ UNS } & \multirow[b]{2}{*}{49} & \multirow[b]{2}{*}{$34,7 \%$} & \multirow[b]{2}{*}{17} & Aprobó & $35,3 \%$ & 6 & \multirow[b]{2}{*}{$65,3 \%$} & \multirow[b]{2}{*}{32} & Aprobó & $43,8 \%$ & 14 \\
\hline & & & & Desaprobó & $64,7 \%$ & 11 & & & Desaprobó & $56,3 \%$ & 18 \\
\hline
\end{tabular}


En el Cuadro 3, puede observarse que la mayoría de los aspirantes estudia con un profesor particular, el cual es pagado por sus familias. No obstante, dicha preparación especial no garantiza que el aspirante pueda aprobar el examen de ingreso. En cuanto a los resultados, se advierte que los que cursan en escuelas de gestión privada tienen mejores resultados que aquellos provenientes de escuelas públicas. Los estudiantes de la escuela primaria de la UNS tienen peores resultados, aunque ellos no necesitaban aprobar para ingresar debido a los privilegios que les otorgan las autoridades. Cabe destacar que, en los últimos años, fueron especialmente preparados por sus maestras de grado, ya que el desempeño en el ingreso era peor que el resto, aun asistiendo al curso de ingreso.

La única alternativa propuesta para compensar estas desigualdades de origen dentro del sistema meritocrático es el "curso de nivelación", el cual es universal para todos los aspirantes, de forma tal que la totalidad de quienes aspiran a ingresar pueden tener una base en común para rendir los exámenes de ingreso y prepararse para el primer año en la escuela que se caracteriza por ser más exigente que el resto de las escuelas secundarias. La universidad debería hacer esfuerzos extra por inclinar la balanza educativa de oportunidades, por ejemplo, ofreciéndoles una preparación extra a quienes cuentan con menos oportunidades educativas por sus orígenes sociales. El antecedente de esta medida en la escuela fue el primer curso brindado en la historia de la ECBC, donde, en 1985, algunos de sus docentes concurrieron a diez escuelas públicas ubicadas en distintos barrios de Bahía Blanca para dictar allí cursos gratuitos de dos meses de duración. El proyecto fue discontinuado por poca convocatoria y siguió brindándose en la escuela (que se encuentra en el centro de la ciudad), pero, en su momento, significó un acercamiento al resto de las instituciones educativas locales.

Usamos el cuadernillo de la UNS todos los años, nos sirve mucho porque vemos qué temas enseñan en la universidad para el ingreso al Ciclo y lo usamos para el siguiente año escolar. Nosotros lo tomamos como un recurso didáctico más. (Directora Primaria Pública, Barrio Colón, 2019)

Asimismo, las demás primarias públicas de la ciudad utilizan anualmente el cuadernillo con ejercicios que entrega la UNS en el curso de nivelación, algunas directoras consultadas mencionaron que son una fuente educativa que contribuye a la actualización de contenidos por parte de sus docentes.

\section{La inclusión proporcional del sorteo}

El sorteo general se presenta como el sistema más justo porque todos los aspirantes tienen igualdad de oportunidades, pero, en la práctica, el azar reproduce proporcionalmente a los grupos sociales que se anotan para el ingreso. Entonces, se produce un sorteo ponderado (Dowlen y Delgado, 2016) por el cual tienen mayores chances aquellos que pertenecen a las clases sociales que registran mayores inscriptos; en el caso de la ECBC, la mayoría son aspirantes de clase media que concurren a escuelas céntricas (tanto públicas como de gestión privada). El sorteo, desde su aplicación parcial a partir del año 2014, por sí mismo no logró la inclusión social de los ingresantes. 
Solo permitió que algunos miembros de la clase mayoritaria puedan ingresar sin necesidad de aprobar las pruebas de lengua y matemática, excluyendo a aquellos pertenecientes a clases de menores recursos que registran cada año menos inscriptos (Anderete Schwal, 2019).

\section{Cuadro 4}

Resultados del sorteo 2021 de aspirantes externos según la gestión de la escuela de origen

\begin{tabular}{|l|c|c|c|c|}
\hline Escuelas & Inscriptos & $\%$ & Sorteados & \% \\
\hline Privadas & 346 & 44,25 & 99 & 47,14 \\
\hline Públicas & 436 & 55,75 & 109 & 51,86 \\
\hline Total & 782 & 100,00 & 208 & 100,00 \\
\hline
\end{tabular}

En el Cuadro 4, se observa el tipo de gestión de las escuelas de origen de los sorteados, el cual se relaciona proporcionalmente con las aquellas de los aspirantes. Aunque, en este caso, los provenientes de escuelas privadas aumentaron su representación después del sorteo. Este dato estadístico ejemplifica los resultados del azar como método de ingreso, el cual replica proporcionalmente el origen educativo registrado en la inscripción y no tiene por sí mismo una función socialmente inclusiva. Asimismo, las escuelas públicas de origen son, en su mayoría, aquellas ubicadas en la delegación Centro de Bahía Blanca (Anderete Schwal, 2019), que se corresponde al nivel socioeconómico medio y alto de la ciudad. En el Cuadro 4, se considera a los 208 aspirantes externos beneficiados por el sorteo. Sin embargo, no todos ellos ingresaron al ciclo lectivo 2021, ya que en este año se registró el mayor número de renuncias de ingresantes en la historia de la ECBC. Quienes renunciaron le dieron su lugar al siguiente aspirante en el listado, según sus últimos tres números del DNI.

Recién en mayo del 2021 se completó el cupo de ingresantes porque hubo renuncias, renuncias y renuncias. Chicos que habían entrado y decidían no acceder al banco. Finalmente, terminaron ingresando 25 chicos que ni siquiera habían hecho el curso de nivelación, se los llamó porque se terminó la lista de sorteados. (Directiva de la ECBC, 2021)

Estas renuncias masivas se vinculan con las resistencias a las clases presenciales por parte del gremio de la universidad, al punto tal de que habían vuelto las clases en las escuelas privadas y públicas provinciales de la ciudad, pero no en las escuelas preuniversitarias de la UNS. ${ }^{\text {viii }}$ La directora del CEMS pertenece a la dirigencia gremial que gobierna a las escuelas y que propone cambios como el sorteo general en el ingreso de estudiantes, ${ }^{i x}$ aunque manteniendo los privilegios de quienes tienen familiares universitarios.

El secretario general de la Asociación de Docentes de la Universidad Nacional del Sur (ADUNS) declaró públicamente que "A esa escuela pública debería accederse como a toda escuela pública del país", ${ }^{x}$ defendiendo el ingreso de estudiantes por sorteo general. Aunque el mismo gremio promueve el ingreso directo de los estudiantes del nivel primario de la UNS y la prioridad de quienes tienen padres universitarios, ya que los hijos de la clase dirigente preuniversitaria suelen concurrir a dichas escuelas y ello garantiza su socialización controlada. Asimismo, el ingreso a las 
demás escuelas públicas del país no es por sorteo general, sino que se sostienen diversos sistemas según las características educativas de cada provincia (Rigal et al., 2019).

\section{Reflexiones finales}

La directora del CEMS dio cuenta de las limitaciones de su autoridad para definir las políticas educativas en el ingreso a la escuela, ya que para cuestionar el elitismo por parte de la ECBC propone el sorteo general —_como una medida igualitaria——, pero, por otro lado, omite mencionar la entrada directa de los estudiantes de la primaria de la UNS quienes, en su mayoría, provienen de familias universitarias. Asimismo, la autoridad no propone políticas educativas activas concretas para atraer al sector social actualmente excluido. Teniendo en cuenta los mecanismos de exclusión social practicados por la escuela, el cambio aislado del sistema de ingreso se convierte en una medida ineficaz para popularizar su composición social. Además, proponen quitar una de las pocas herramientas de inclusión educativa, que es el curso de nivelación destinado a todos los aspirantes y cuyo contenido no se reduce solamente al ingreso, sino a nivelar la base educativa de los estudiantes (sean o no ingresantes). Finalmente, el Consejo Superior Universitario trató el tema y decidió mantener el mencionado curso en forma virtual y asincrónica, debido a las diversas condiciones de acceso a internet de las familias. También se resolvió que aquellos que por cuestiones económicas no puedan acceder a los contenidos digitales, serán ayudados por la UNS en todo lo que necesiten. Tal como lo hace con sus estudiantes de grado que tienen dificultades para cursar en las clases virtuales dictadas durante la pandemia. De los 782 estudiantes inscriptos para el ingreso 2021, solamente uno solicitó ayuda para acceder digitalmente a los contenidos del curso de ingreso. En este caso, la UNS lo proveyó de una Tablet para que pueda estudiar y prepararse para el ingreso al Ciclo Básico.

En el caso de Bahía Blanca (y las demás ciudades de Argentina con escuelas preuniversitarias tradicionales), no se puede desconocer que el paso del tiempo generó un habitus en determinados grupos que aspiran a pertenecer a dicha institución y, por contrapartida, un alejamiento de los que históricamente se vieron excluidos y continuaron por sus propios circuitos educativos. Por tal motivo, si la UNS pretende incluir a estos sectores, debe realizar un cambio estructural orientado a sumar a este nuevo público.

Dado el estado de situación actual, el mecanismo de ingreso a la escuela por sorteo general termina favoreciendo a las familias de clase media que representan a la mayoría de los candidatos (Anderete Schwal, 2019). Esta medida, implementada en forma aislada, no cumple con su objetivo manifiesto de reducir el rol que las escuelas preuniversitarias de la UNS cumplen en la reproducción de la desigualdad social en el contexto educativo local. De tal forma, no se logra disminuir la desigualdad socioeconómica en el ingreso atribuida a la meritocracia, sino que se acentúa reproduciendo proporcionalmente la desigualdad social entre sus aspirantes.

Para efectivamente favorecer la inclusión social a través del ingreso a la escuela estudiada, las medidas debieran versar sobre la incorporación de sectores sociales relegados mediante políticas educativas activas por parte de la escuela y de la Universidad Nacional a 
cargo, tal como realizan las Nuevas Escuelas Preuniversitarias de las Universidades Nacionales. Algunas de tales medidas podrían ser: acercarse a los distintos barrios de la ciudad; incrementar la interacción con las demás escuelas públicas provinciales; ofrecer becas a quienes no pueden trasladarse al centro o requieren asistencia para el comedor o los materiales educativos; reservar vacantes exclusivas para ciertos sectores socioeconómicos o estudiantes con discapacidad; implementar estrategias de difusión más abarcadoras; entre otras. La incorporación a un curso de ingreso extendido para un cupo especial por cuestiones socioeconómicas (destinado a los provenientes de escuelas públicas de la periferia, que es la población socialmente excluida actualmente por la escuela), así como otorgar un cupo por discapacidad, serían un buen punto de partida para desarrollar una institución más inclusiva. Asimismo, no parece apropiado justificar la exclusión de cierto grupo social suponiendo que los alumnos no podrán cumplir con las expectativas académicas de la institución; por el contrario, se deberían implementar medidas compensatorias antes y después del ingreso para asegurar su retención.

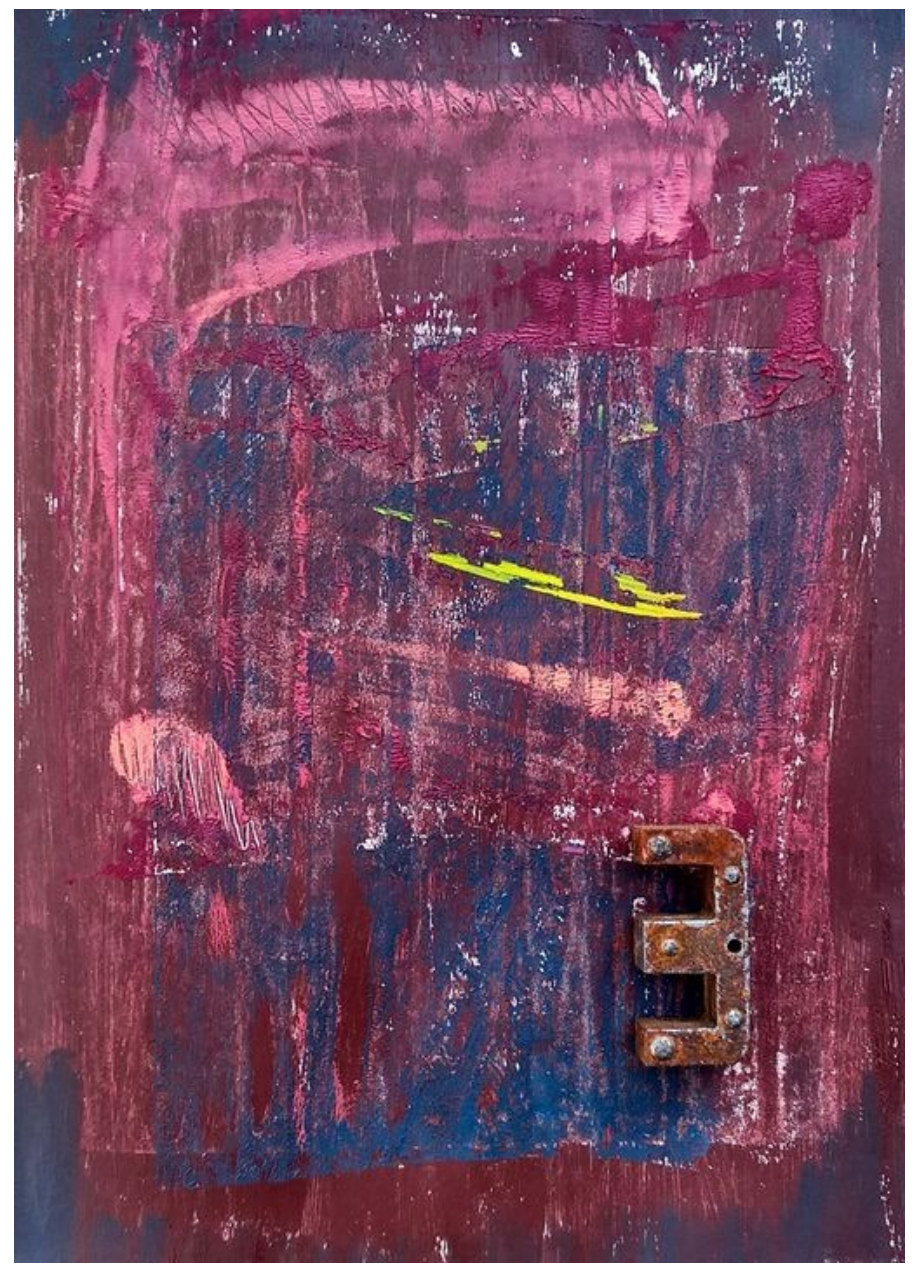

Bayernleverkusen, acrílico. Matias Sapegno 
i https://www.lanueva.com/nota/2020-7-2-6-30-12-la-directora-del-cems-sobre-el-ingreso-al-ciclosiempre-dio-un-sesgo-de-elite

ii Debate Res. 696/13 CSU UNS. https://st02.uns.edu.ar/contenidos/documentos/45_AP_0_162.pdf

iii http://www.uns.edu.ar/noticias/2018/4585

iv http://sabato.unicen.edu.ar/node/15

$\checkmark \quad$ http://noticias.unsam.edu.ar/2020/3/12/de-la-escuela-a-los-barrios-no-solo-te-esperamos-tambiensalimos-a-buscarte/

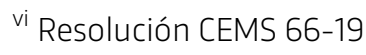

vii CSU-189/2020

viii https://bahia.telefe.com/locales/nueva-marcha-de-padres-y-alumnos-por-el-regreso-a-lapresencialidad-en-las-emuns/

${ }^{\text {ix }}$ http://www.frenteacano.com.ar/noticia/222506

x https://www.lanueva.com/nota/2020-7-8-6-30-10-zaninelli-sobre-el-ingreso-al-ciclo-deberia-accedersecomo-a-toda-escuela-publica

\section{Bibliografía}

Anderete Schwal, M. (2019). Efectos del sorteo en una escuela secundaria preuniversitaria. Revista Espacios En Blanco, 30(1), 159-177. UNICEN.

Anderete Schwal, M. (2021). Las Nuevas Escuelas Secundarias Preuniversitarias (2013-2020): La inclusión como paradigma. Entramados: educación y sociedad, 9(8). UNMdP.

Antico, D., Costanzo, P. y Dettorre, L. (2019). Construyendo nuevos escenarios educativos. Desafíos para la configuración de nuevos roles docentes en las escuelas preuniversitarias del conurbano bonaerense. Sociales y Virtuales, 6(6).

Aguilar, O. (2011). Dinero, educación y moral: el cierre social de la élite tradicional chilena. En A. Joinant y P. Guell (Eds.), Notables, tecnócratas y mandarines. Elementos de la sociología de las élites en Chile (1990-2010). Salesianos impresores.

Bernstein, B. (1988). Poder, educación y conciencia: sociología de la transmisión cultural. Cide. Bourdieu, P. y Passeron, J. C. (2003). Los herederos. Los estudiantes y la cultura. Siglo XXI.

Chávez, M. (2017). Jóvenes entre el centro y la periferia de la ciudad, del estado y de la academia. CiudaDanías, 7(6), 1-18.

Corbetta, P. (2003). Metodología y técnica de la Investigación Social. McGraw Hill.

De Imaz, J. (1964). Los que mandan. EUDEBA.

Di Piero, E. (2015). ¿Mérito y azar? Nociones de justicia distributiva y selección soft: el caso de una escuela secundaria tradicional frente al mandato inclusor. Propuesta educativa, (43),152-154.

Di Piero, E. (2016). Igualitarismo, mérito y nociones de justicia en torno al método de admisión a una escuela secundaria universitaria. Archivos de Ciencias de la Educación, 10(10).

Dowlen, O. y Delgado, J. (2016). El sorteo en Política. Cómo pensarlo y cómo ponerlo en la práctica. Efialtes. Dubet, F. (2012). Repensar la justicia social. Contra el mito de la igualdad de oportunidades. Siglo XXI. 
Dussel, I. (2009). La cultura escolar en contextos fragmentados: notas para pensar sobre qué puede una escuela. Mimeo.

Dussel, I. (2014). ¿Qué lugar tiene la escuela media en la producción de la desigualdad? Elementos para el debate. Archivos Analíticos de Políticas Educativas, 22(43).

Filmus, D. (1996). Estado, sociedad y educación en la Argentina de fin de siglo: proceso y desafíos. Troquel. Garino, M. D. (2013). Tensiones y desafíos en torno a la masificación de la escuela secundaria. Reflexiones a partir de una propuesta educativa en la ciudad de Neuquén. Revista de la Escuela de Ciencias de la Educación, (8).

Gil, J. M. (2019). Paradojas excluyentes del "lenguaje inclusivo" en torno al uso del morfema flexivo -e. Segundas Jornadas Nacionales y Primeras Internacionales de Estudios Lingüísticos. La reflexión lingüística: de la investigación al aula. Mendoza.

Heredia, M. (2005). La sociología en las alturas. Aproximaciones al estudio de las clases/élites dominantes en la Argentina. Apuntes de investigación del CECYP, IX (10).

Krüger, N. (2019). La segregación por nivel socioeconómico como dimensión de la exclusión educativa: 15 años de evolución en América Latina. Archivos Analíticos de Políticas Educativas, 27(8).

Landivar, T. (2019). Las escuelas secundarias de Universidades Nacionales Argentinas: datos y reflexiones. Editorial Unicen.

Langer, E. y Esses, J. (2019). La salida es por arriba. Una historia de juventud, pobreza y educación. Grupo Editor universitario.

Méndez, A. (2013). El Colegio: la formación de una élite meritocrática en el Nacional Buenos Aires. Ed. Sudamericana.

Méndez, A. (2015). Lenguajes, retóricas y repertorios de egresados de un colegio público "de elite". ProPosições, 26(2), 119-139.

Rigal, J., Schoo, S. y Ambao, C. (2019). El ingreso a la escuela secundaria. Un análisis de los sistemas de distribución de vacantes en Argentina. Serie Informes de Investigación No 10. Área de Investigación y Seguimiento de Programas. Dirección de Información y Estadística Educativa (DIEE).

Veleda, C. (2014). Regulación estatal y segregación educativa en la Provincia de Buenos Aires. CIPPEC. UNICEF. Veleda, C., Rivas, A. y Mezzadra, F. (2011). La construcción de la justicia educativa. Criterios de redistribución y reconocimiento para la educación argentina. CIPPEC-UNICEF. 